\section{Pancytopenia in a Patient with Psoriatic Arthritis Treated with Methotrexate and Concomitant Lithium}

\section{To the Editor:}

We report a case of pancytopenia in a patient with psoriatic arthritis (PsA) treated with methotrexate (MTX) and concomitant lithium. Our index patient was a 55-year-old white female who had PsA since age 28. She had arthritis mutilans. Her disease was well controlled while being treated with MTX $15 \mathrm{mg}$ weekly together with folic acid $10 \mathrm{mg}$ weekly for many years. She was not receiving any nonsteroidal antiinflammatory drugs because her arthritis was well controlled with disease-modifying agents. Both her psoriasis as well as the PsA were well managed with the above medication. She had been followed up regularly with liver function tests and complete blood count tests every 3 months with no toxicity from the MTX. In March of 2012, she presented to her general practitioner with symptoms suggesting bipolar disorder. Her general practitioner then commenced treatment with lithium $400 \mathrm{mg}$ daily.

Two months after the commencement of lithium, the patient presented with epistaxis and a complete blood count showing severe pancytopenia. Her hemoglobin was $6.5 \mathrm{~g} / \mathrm{dl}$, white cell count was $2.07 \times 10^{9} / 1$ with an absolute neutropenia, and her platelet count was $25 \times 10^{9} / 1$. Her renal function was normal. Lithium levels measured upon hospital admission were within the therapeutic range. MTX levels were not taken upon admission. She was treated with blood transfusions, granulocyte-macrophage colony-stimulating factor (GM-CSF), as well as platelet transfusions and folinic acid. Her course in the hospital was uneventful and she was discharged 2 weeks later with a full recovery of her bone marrow.

In June of 2012, the patient's PsA flared. She presented with a tender joint count of 10 and a swollen joint count of 14. During the period between March and June 2012, the patient was not receiving MTX. A repeat complete blood count and liver function tests were normal and MTX was reintroduced at a dose of $15 \mathrm{mg}$ weekly together with folic acid $10 \mathrm{mg}$ weekly. All her other medication was stopped.

The patient remained well until January 2014 when she was readmitted to hospital with septicemia. During the investigation of the septicemia, it was found that she had a severe pancytopenia with a hemoglobin of 4.6 $\mathrm{g} / \mathrm{dl}$, a white cell count of $0.9 \times 10^{9} / 1$, and a platelet count of $4 \times 10^{9} / 1$. She had an absolute neutropenia. Urea and electrolytes showed her to be in acute renal failure with an estimated glomerular filtration rate of 10 $\mathrm{ml} / \mathrm{min}$. Because the patient was in renal failure, MTX and lithium levels were not taken prior to the commencement of the dialysis. It was then brought to our attention that 2 months prior to presentation, the patient's general practitioner had reintroduced lithium at a dose of $400 \mathrm{mg}$ daily. From March 2012 to November 2013, the patient was not receiving lithium and had no hepatotoxicity or myelosuppression from the MTX.

During the next few weeks, the patient was in the intensive care unit with broad-spectrum antibiotics, red cell transfusions, platelet transfusions, folinic acid, and GM-CSF, together with hemodialysis. The patient's blood culture developed Klebsiella pneumonia. Sensitivities were obtained from the microbiologist and target therapy was introduced. Her procalcitonin levels were $37.3 \mathrm{ng} / \mathrm{ml}$ (reference range for our laboratory was $<0.5$ ).
The patient made an uneventful recovery and was discharged from the hospital with normal blood count and liver function tests.

This patient was introduced to lithium at 2 different times, 2 years apart, and both times developed severe myelosuppression 2 months later.

We have done an extensive literature search through PubMed and found no documented evidence that lithium can cause the myelosuppressive effects of MTX. Lithium has been used to treat neutropenia in 6 patients with mixed results ${ }^{1}$. Despite having normal lithium levels, all 6 patients developed toxicity from lithium and the response was transient. Although there was a temporal relationship between the introduction of lithium and the development of myelosuppression in this patient on both occasions, MTX may also cause myelosuppression. The exact mechanisms by which the lithium caused the myelosuppression is not clear. It increases protective proteins such as brain-derived neurotrophic factor and B cell lymphoma 2, and reduces apoptotic processes through inhibition of glycogen synthase kinase 3 and autophagy ${ }^{2,3}$. Overall, it is clear that the processes through which the therapeutic actions of lithium work are sophisticated and most likely interrelated. However, how it caused myelosuppression in our patient may be multifactorial. Nephrotoxicity from lithium therapy leading to decreased excretion of MTX and its metabolites may be responsible for the myelosuppression in this patient. Unfortunately, MTX levels are not routinely measured in rheumatological patients treated with low-dose MTX. To our knowledge, ours is the first documented case of such an occurrence.

AJESH B. MAHARAJ, MB, BS, IMS, BHU (Varanasi), H.Dip.Int.Med (SA), FCP (SA), Certificate in Rheumatology (SA), Division of Clinical Immunology and Rheumatology, Academic Medical Center/University of Amsterdam, Amsterdam, the Netherlands, and Prince Mshiyeni Memorial Hospital, Department of Internal Medicine, Nelson R. Mandela School of Medicine, University of KwaZulu Natal, Durban, South Africa; VINOD CHANDRAN, MB, BS, MD, DM, PhD, Division of Rheumatology, University of Toronto, Toronto Western Hospital, Toronto, Ontario, Canada. Address correspondence to Dr. A. Maharaj, Academic Medical Center, Immunology and Rheumatology, University of Amsterdam, Amsterdam, 1100 DD Amsterd, the Netherlands. E-mail: maharaja30@ukzn.ac.za

\section{REFERENCES}

1. Mant MJ, Akabutu JJ, Herbert FA. Lithium carbonate therapy in severe Felty's syndrome. Benefits, toxicity, and granulocyte function. Arch Intern Med 1986;146:277-80.

2. Davies SP, Reddy H, Caivano M, Cohen P. Specificity and mechanism of action of some commonly used protein kinase inhibitors. Biochem J 2000;351:95-105.

3. Jope RS. Anti-bipolar therapy: mechanism of action of lithium. Mol Psychiatry 1999;4:117-28.

J Rheumatol 2015;42:3; doi:10.3899/jrheum.141022 